\title{
Arbor
}

\section{Una visión de género en el Área de Recursos Naturales}

M. Teresa Tellería

Arbor CLXXII, 679-680 (Julio-Agosto 2002), 641-654 pp.

Se me pide que escriba, dentro de este monográfico dedicado a una "visión de género» en el CSIC, acerca de la situación concreta en el área de Recursos Naturales. El encargo que se me hace es para mí doblemente complicado. Por un lado, he de escribir sobre algo de lo que únicamente tengo una vaga opinión personal, a buen seguro sesgada, fruto de mi particular experiencia y de mi trayectoria vital lo que, para el caso que nos ocupa, tampoco es tan importe pues es la opinión de una entre muchas. Por otro, es un tema sumamente complejo y abordarlo con el rigor metodológico suficiente, como para poder extraer conclusiones que tengan algún interés general, es algo que se escapa, desde todo punto de vista, a mis conocimientos y a los datos de los que dispongo. De tal modo que lo que a continuación viene es fruto de una opinión particular aunque, para emitirla, he procurado documentarme previamente. Los datos objetivos, relativos a la situación concreta de las investigadoras del CSIC, los he tomado, entre otras fuentes, del «Estudio sobre mujeres investigadoras del CSIC» [http:/ / www.csic.es] y, los relativos al área, del último Plan de Actuación del CSIC, 2000-2004. He consultado, así mismo, otras fuentes relativas a la plantilla actual de personal investigador del Organismo.

\section{El área de Recursos Naturales, sus características}

El área de Recursos Naturales se define, en el Plan de Actuación del CSIC, como de temática compleja y heterogeneidad disciplinaria. Un re- 
paso por las líneas de investigación que se desarrollan en los institutos que la integran nos lleva desde la Geoquímica a los estudios concretos de Fauna y Flora con la taxonomía descriptiva, la sistemática molecular y la paleontología como estandartes, y por un camino que pasa por la Evolución y Dinámica de la Litosfera, las Ciencias del Suelo y Procesos Superficiales, Estructura y Procesos Oceánicos se llega a los Sistemas Litorales, la Acuicultura y Pesquerías. La Ecología Evolutiva y del Comportamiento y, de nuevo, la Ecología esta vez de poblaciones cierran la tabla. Cada epígrafe principal abarca, a su vez, un acervo temático tan rico como variado lo que se concreta en más de treinta grandes líneas de investigación.

Tal heterogeneidad disciplinaria se reparte, según la referida memoria, en 18 Institutos (ver apéndice 1), aunque este número llega a 25 si lo que consultamos es la información de la web del CSIC ${ }^{1}$. En cualquier caso, un elevado número de centros repartidos por toda la geografía española. Además, el área dispone de algunas grandes instalaciones como estaciones de campo, buques oceanográficos o colecciones de organismos, estas últimas punto de referencia obligado en los estudios de biodiversidad.

Por su producción científica, según la misma fuente que se refiere al anterior quinquenio (1995-1999), el área de Recursos Naturales ocupa el primer lugar, entre las del CSIC, si hablamos del número total de publicaciones producidas: 7.390 entre artículos publicados en revistas incluidas en el SCI (3.539), no incluidas en el SCI (2.165), así como libros y asimilados (1.686). Si comparamos estos datos, al menos parcialmente, con los de las otras áreas podemos decir que: en lo referente a la publicación en revistas incluidas en el SCI, el área de Recursos Naturales ocupa el tercer lugar, tras Ciencia \& Tecnología de Materiales (con 4.517 artículos) y Biología \& Biomedicina (con 4.101 artículos) que se colocan en primer y segundo lugar respectivamente; tras Recursos Naturales, Ciencia \& Tecnologías Químicas (con 3.469 artículos) y Físicas (con 3.199). Si en vez de los artículos publicados en revistas incluidas en el SCI, tomamos como punto de referencia las que no lo están, entonces Recursos Naturales pasa de nuevo a la cabeza, seguida por el área de Humanidades con un número total de publicaciones en este tipo de revistas de 1.621. El orden se invierte entre estas dos áreas cuando hablamos de libros o asimilados. Humanidades pasa a la cabeza, con 2.291 volúmenes editados, seguida de nuestra área que, como hemos dicho, acumula un total de 1.686.

Pasemos ahora de las publicaciones a otro de los parámetros que se utilizan en este tipo de evaluaciones: el económico. Más concretamente aquel que se refiere a los recursos captados por cada una de las áreas y 
también referido al quinquenio 1995-1999. El área de Recursos Naturales ocupa, en este 'ranking', el segundo lugar con 9.011.406 millones de pesetas, detrás de Biología \& Biomedicina que es la que se sitúa en cabeza con 16.265.891 millones; tras Recursos Naturales, las áreas de Ciencias \& Tecnologías Físicas, Químicas y de Materiales forman un grupo bastante homogéneo con 7.750.882, 7.460.974 y 7.456.329 millones respectivamente.

Todos estos datos, están referidos a una plantilla de personal investigador que, a finales de $1999^{2}$, era de 2.085 investigadores. El área de Recursos Naturales que tenía 294 ocupaba el tercer lugar, tras la de Biología \& Biomedicina y la de Ciencia \& Tecnología de Materiales con 334 y 308 investigadores respectivamente.

\section{Análisis cuantitativo de género en el área}

Los datos de los que se dispone para el análisis que a continuación se realiza proceden, como hemos dicho en la introducción, del estudio sobre mujeres investigadoras publicado en la web del CSIC [http://www.csic.es/ hispano/mujeres/ciencia.htm]. Según estos datos son las áreas de Ciencia $\&$ Tecnologías Físicas, con un 19,9\% y la de Recursos Naturales con un $23,5 \%$, las que tienen un porcentaje más bajo de investigadoras en su plantilla. Distan mucho estas cifras de las que ocupan los puestos más altos de la tabla: Ciencia \& Tecnología de los Alimentos con un $39,8 \%$ y Ciencias Agrarias con un 37,7\%. El área de Recursos Naturales está también bastante alejada de la media general del Organismo que se sitúa en un $30,9 \%$ de investigadoras.

Un análisis por escalas dentro del área refleja que las mujeres son el $22 \%$ de los integrantes de la escala de Profesores de Investigación, el 20\% de los Investigadores Científicos y el $26 \%$ de los Científicos Titulares. Quizá no esté de más añadir que el primer porcentaje lo es sobre un total de 37 individuos, el segundo de 81 y el tercero de 188, lo que significa que, en junio de 2001, el número de Profesoras de Investigación en el área era de 8, el de Investigadoras 16 y el de Científicas Titulares 48 (ver gráfica $1^{3}$ ). Si comparamos estos datos con los generales del Organismo, el área está muy por encima de la media, que se sitúa en el 1.3,3\%, en lo relativo a la escala de Profesores de Investigación y por debajo en las de Investigadores Científicos donde la media está en el $26 \%$ y de Científicos Titulares que se sitúa en el 37\%.Para completar la descripción cuantitativa del área añadiremos que de los 18 Institutos que la integran solo cuatro, según los datos de los que dispongo, están dirigidos por mujeres, 
lo que supone el $22 \%$, un porcentaje algo inferior al de la media del Organismo que se sitúa en el $25 \%$.

GRÁfICA 1. Muestra la distribución, por categorías y género, en el área de Recursos Naturales. Científicos titulares (negro); investigadores científicos (rayas en diagonal); profesores de investigación (rayas horizontales)

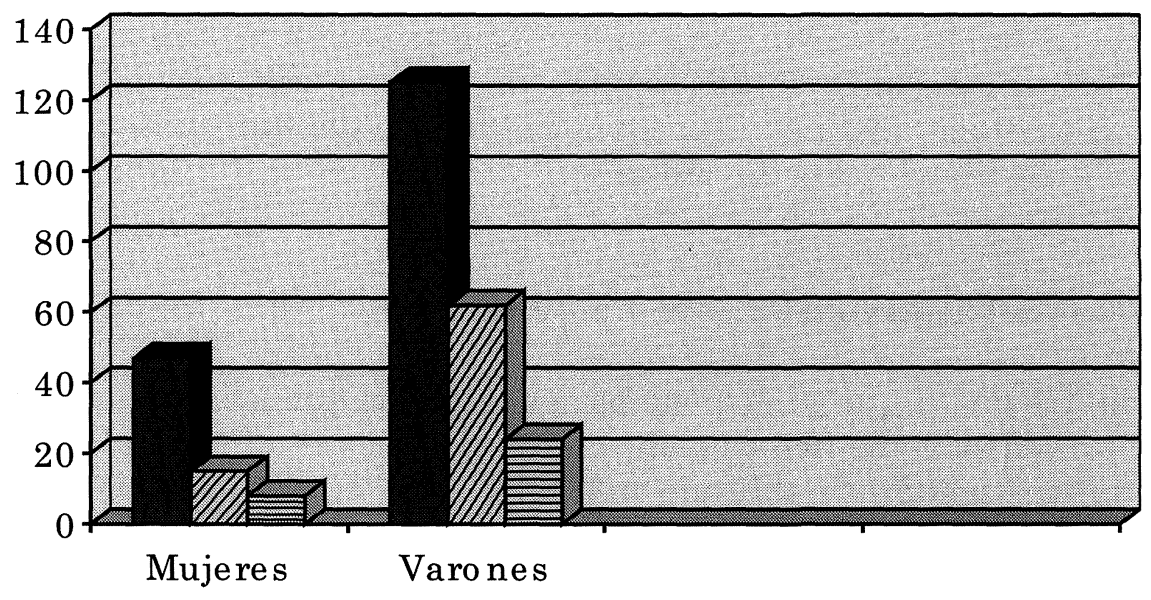

\section{Evolución de la plantilla investigadora en Recursos Naturales}

Tras estos análisis comparativos con otras áreas vamos a tratar de describir, de modo detallado, cual es la situación concreta dentro del área que nos ocupa. Para ello utilizaremos un mayor número de datos que proceden también del CSIC y que son los relativos al personal investigador hasta 1999. Es probable, por tanto, que algún dato difiera ligeramente de los utilizados en la comparación anterior. No obstante las diferencias no van a ser nunca significativas.

El acceso a la escala de Científicos Titulares ha venido marcado en el área, como en otras del CSIC, por la política científica del país y la del propio Organismo.

La mayoría del personal investigador que en este momento constituye la plantilla del área ha accedido a la carrera científica a través de la escala de Científicos Titulares y lo ha hecho, fundamentalmente, en tres etapas.

La primera es la que va desde 1970 a 1981 . Según se refleja en la gráfica 2 ingresaron en ese periodo un total de 84 Científicos Titulares de los que un $23 \%$ eran mujeres (20 frente a 64 ). 


\section{Una visión de género en el Área de Recursos Naturales}

GRÁfICA 2. Acceso a la escala de científico titulares por convocatorias, en el área de Recursos Naturales. Columna oscura: varones. Columna clara: mujeres

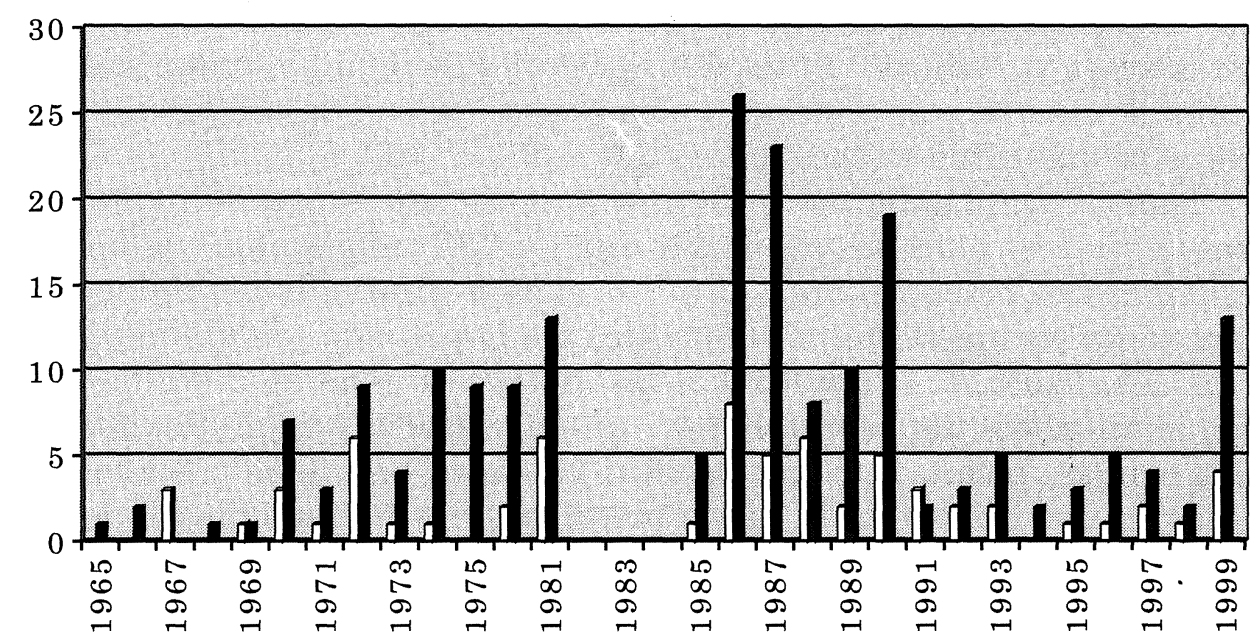

Entre los años 1982-1984, ambos inclusive, hay un paréntesis en el que no se produce ninguna nueva incorporación. A partir de 1985 se inicia una época de expansión que llega hasta 1990. Se cubren un total de 118 plazas de las que 91 son ocupadas por varones y 27 por mujeres, lo que supone un $22,88 \%$. Son especialmente llamativos, por el número de plazas convocadas, los años 1986 y 1987 con 34 y 28 plazas respectivamente de las que un $23,5 \%$ y un $17 \%$ fueron ocupadas por mujeres. Algo similar ocurre el año 1990 en que se cubren un total de 24 plazas de las que 5 (el 20,8\%) son ocupadas por mujeres.

Entre los años 1991 y 1998 vuelve una época de recesión en la incorporación de nuevos científicos a la plantilla del área. Se convocan un total de 38 plazas y de ellas 12 (el 31,57\%) son ocupadas por Científicas Titulares. Finalmente, en el año 99 se detecta un nuevo incremento en el número de plazas y el porcentaje de mujeres baja a un $23,53 \%$ de las plazas lo que nos coloca en los tantos por ciento de los años setenta.

Para poder analizar en detalle estos datos sería importante saber el número de becarias / becarios tanto predoctorales como postdoctorales que acceden al área $y$, de éstos, cuantos llegan a presentarse a las oposiciones.

Sería también importante poder disponer, para este caso y los que a continuación vienen, de los datos necesarios para analizar la proporción 
de candidatos presentados/plazas cubiertas por varones y mujeres en cada una de las escalas y la evolución de estos datos en el tiempo. Esto nos permitiría hacer un examen más preciso de la situación y plantear, sobre la base de datos cuantificables, alguna hipótesis que ayude a explicar la curiosa situación que se presenta, bastante atípica con relación a otras áreas y que no se ajusta a los patrones del CSIC en particular ni del mundo científico en general, donde la presencia de las mujeres en las categorías más altas es poco menos que testimonial.

Pasamos seguidamente a examinar el acceso a la escala de Investigadores Científicos pues nos puede dar una idea de como evoluciona la promoción interna dentro al área. Como en el caso anterior, hay también diferentes etapas marcadas por la política del Organismo. Según los datos de los que dispongo, la plantilla actual de Investigadores Científicos se ha promocionado fundamentalmente en dos periodos (ver gráfica 3 ).

El primero a comienzos de los años 70, entre 1971 y 1973 ambos inclusive, en que ascienden 22 de los Investigadores hoy en activo, y lo hacen según la siguiente proporción: 8 mujeres frente a 14 varones. Un periodo intermedio, que va desde 1974 hasta 1984, con un número de promociones mínimo: cambian de escala un total de 6 investigadores, todos varones; y el tercero que comienza en el año 1986 y llega hasta 1993. El número de accesos a la escala de Investigadores Científicos en esta etapa es elevado, un total de 72 , con un número muy reducido de mujeres, apenas un 15,27\% (es decir 11 mujeres frente a 61 hombres).

GráfICA 3. Acceso a la escala de Investigadores Científicos por años, en el área de Recursos Naturales. Columna oscura: varones. Columna clara: mujeres

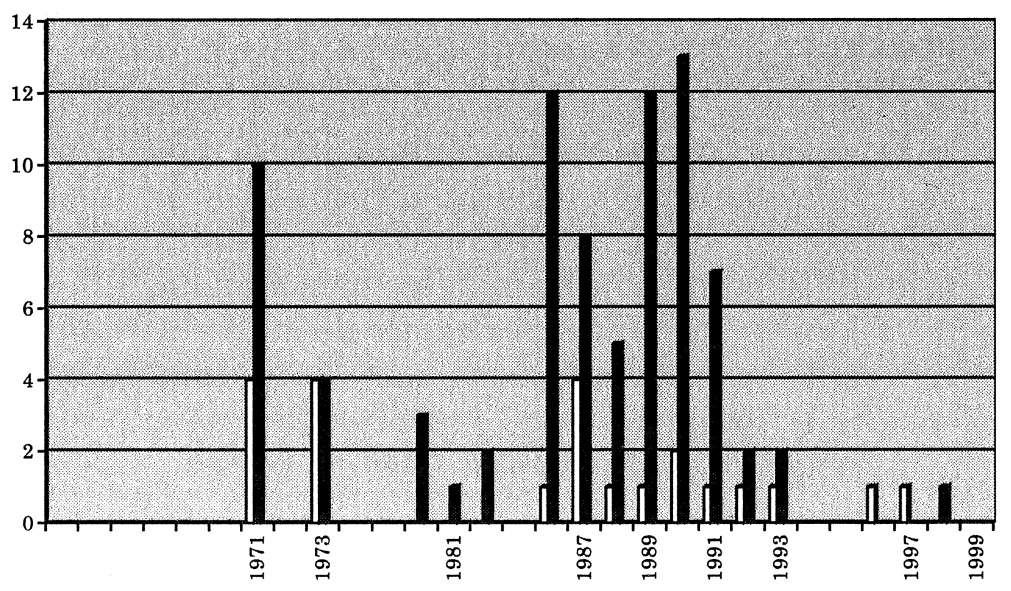




\section{Una visión de género en el Área de Recursos Naturales}

Se puede comentar además que hay también una significativa diferencia de género en el número de Investigadores Científicos que han accedido a esa escala directamente. De los actualmente en activo, los varones son 10 frente a las mujeres que son únicamente 2. En ambos casos una cantidad mínima, pero bastante inferior en las mujeres: un $2,59 \%$ frente a un $10 \%$.

Llegamos, por último, a la escala de Profesores de Investigación donde, lo mismo que sucede en otras áreas, la promoción es a cuentagotas. En el área de Recursos Naturales ésta tiene lugar, sobre todo, en el periodo que va desde 1984 hasta 1993. En esta etapa (ver gráfica 4) el número total de plazas es de 29 de las que 5 son ocupadas por mujeres (un 17, $24 \%$ ). En los últimos años ha habido un incremento - no dispongo de esos datos pero se deducen de los referidos en el Estudio sobre las mujeres investigadoras en el CSIC - hasta llegar a un $22 \%$ del total de los de la plantilla. Tanto en un caso como en otro, el porcentaje está por encima de la media del Organismo

Gráfica 4. Acceso a la escala de Profesores de Investigación por años, en el área de Recursos Naturales. Columna oscura: varones.

Columna clara: mujeres

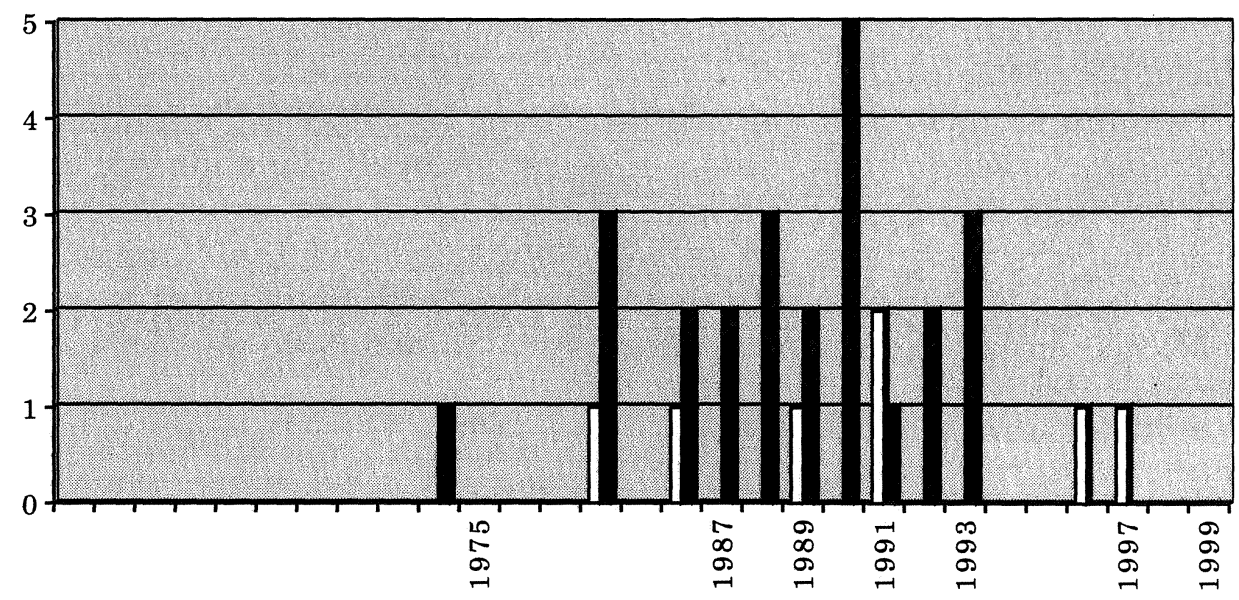

\section{Los criterios de evaluación}

No descubro nada nuevo a estas alturas si digo que hay una clara discriminación de género en la Ciencia. Los datos objetivos así lo prueban. Un problema general que se escapa a la escala del área, a la del CSIC, a 
la de nuestro país y a la de los de nuestro entorno geográfico y cultural y que se extiende, en mayor o menor medida, por todas las ramas del saber y líneas de investigación. Siempre el porcentaje de investigadoras es menor y sus posibilidades de promoción inferiores. Se han buscado causas de todo tipo y probablemente todas colaboren a que la situación sea la que es. Se habla del antagonismo entre la vida familiar y la profesional, de los efectos de la socialización de género, de la asignación de roles donde el papel tradicional de la mujer es el de secundaria. Son causas generales que afectan a la mujer en todos los campos y a los que no escapa, por supuesto, el de la actividad científica. Pero además en el mundo científico concurren una serie de circunstancias que es preciso analizar con detalle.

La Ciencia ha sido tradicionalmente una actividad androcéntrica, un coto masculino en el que han entrado un número muy escaso, casi testimonial, de mujeres. Solo en los últimos años la mujer está irrumpiendo en el mundo científico y la situación comienza à cambiar. Los obstáculos han pasado de ser patentes a sutiles y se ha dicho, no sin razón, que más que un número pequeño de grandes desventajas las mujeres debemos hoy en día hacer frente, en el campo científico, a un número muy grande de pequeños y a veces imperceptibles obstáculos. La discriminación no es ahora, como en otro tiempo, abiertamente beligerante y activa pero si hay una discriminación inconsciente, basada en un sistema sesgado de selección y promoción.

Hay unanimidad a la hora de establecer los criterios generales que deben regir la selección del personal investigador. Nadie duda que el criterio fundamental es el de la calidad científica de los candidatos; éste debe imperar sobre todos los demás. Lo que no está tan claro son los parámetros que se deben utilizar para evaluar esa calidad y, es muy probable, que en la aceptación casi dogmática de una serie de ellos esté la raíz de la discriminación.

¿Cómo determinar la calidad de un científico dado? Parece que la respuesta habría que ir a buscarla por los derroteros del interés y novedad de sus objetivos, en el rigor de su trabajo, en la excelencia de sus resultados y, en algún valor algo menos tangible y general, como la honradez para consigo mismo y con su trabajo. Es curioso que esta última sea una cualidad más apreciada por las científicas que por los científicos, un $24 \%$ frente a un 9\% (cf. Holton in http://www.sciam.com/explorations/ 1998/051898women/gender.hrml]. Pero la calidad en realidad se determina sobre la base de otros parámetros de valoración relacionados más, con el modo de enfrentarse a los problemas, la manera de resolverlos y, sobre todo, como abordar la difusión de los resultados, que con la calidad 


\section{Una visión de género en el Área de Recursos Naturales}

propiamente dicha. En suma, están más relacionados con el cómo que con el qué.

Hay una visión estereotipada del científico y el modelo es claramente masculino. En el modelo imperante, forjado a lo largo del tiempo, cualidades como la competitividad y la rivalidad que suelen traducirse en un nivel más alto de productividad, con un mayor número de trabajos publicados, juegan un papel muy relevante. Un buen número de publicaciones en revistas de alto impacto es el pasaporte seguro para una rápida promoción. Por el contrario, un menor número de publicaciones, aunque de ellas se puedan extraer conclusiones más interesantes, publicadas en revistas con menos repercusión pueden retardar o incluso colapsar una carrera.

No quiere esto decir que no sea posible tener éxito en el campo de la investigación científica para aquellas personas menos competitivas, solo quiere decir que el sistema recompensa a aquellos individuos con este tipo de rasgos.

Es, este modo de concebir las cosas que por razones culturales se adapta mejor a la psicología masculina que a la femenina, lo que perjudica seriamente a las mujeres. La discriminación, por tanto, hay que pensar que no es ni consciente ni intencionada, sucede que se aplican criterios de selección basados en estereotipos, con frecuencia, irrelevantes para determinar la competencia científica de los individuos, pero que afectan negativamente a la promoción de las mujeres.

\section{La desigual situación del área de Recursos Naturales}

Recapitulemos. El área de Recursos Naturales tiene en su plantilla uno de los porcentajes más bajos de investigadoras de todo el Organismo. La distribución de las mismas por escalas es francamente atípica, con un tanto por ciento por encima de la media en la escala más alta, Profesores de Investigación, y bastante por debajo en la de Investigadores y Científicos Titulares.

La desproporción de género está ya en el punto de partida, a la hora del ingreso en la escala de Científicos Titulares. En aquellos periodos en que el número de plazas ha sido mayor y, por tanto, los porcentajes más significativos -como por ejemplo los años 86,87 y 90 - el número de mujeres que ha ingresado en esta escala apenas si supera el $20 \%$.

La estructura de la plantilla en el área es piramidal pero, si comparamos por separado la plantilla de varones y mujeres, vemos que en el caso de los primeros el 59\% son Científicos Titulares, el 29,38\% Investi- 
gadores y el 11,37\% son Profesores de Investigación, mientras que en el de las mujeres es porcentualmente superior el número de Científicas Titulares, con un $67,14 \%$, en detrimento de las Investigadoras Científicas que son el $21,42 \%$ y está prácticamente igualado en la escala más alta de Profesores de Investigación. Bien es verdad que en este último caso los porcentajes lo son sobre un número muy bajo de datos ( 24 hombres frente a 8 mujeres) lo que puede hacerlos poco significativos.

Hemos dicho también que el área de Recursos Naturales es competitiva y heterogénea y hemos hecho, así mismo, algunas consideraciones sobre la investigación científica como una actividad tradicionalmente androcéntrica; se trata ahora con todos estos factores y algunos más de lanzar alguna hipótesis que ayude a explicar la, en parte, atípica situación de las mujeres investigadoras en nuestra área.

Las estadísticas del Ministerio de Educación, Cultura y Deporte avalan la total equiparación entre universitarios y universitarias. Según los últimos datos [http://www.mec.es/mec/estadistica/p_estadist.html], relativos al curso 2000-2001, el porcentaje de alumnos en todas las universidades españolas es de un $52,68 \%$ de mujeres frente a un $47,32 \%$ de varones que, en los estudios de tercer ciclo, se sitúa en un 50,34\% para las primeras frente al $49,66 \%$ para los segundos.

En el mundo académico y universitario los parámetros de valoración están basados en criterios claros: es necesario probar que se tienen una serie de conocimientos previamente fijados por un programa. Mediante un sistema, más o menos continuo de evaluaciones, se llega a la consecución del objetivo: acreditar una competencia, certificada por una titulación, que ha de permitir a los candidatos desarrollar una determinada actividad. El sistema es sencillo y no está sujeto, en principio y de un modo muy general, a ningún estereotipo. Un análisis a fondo de la situación, realizado bajo el prisma de las diferentes ramas del conocimiento, probablemente nos daría una mayor riqueza de matices a este asunto, pero creo que eso es, en este momento, irrelevante para el caso que nos ocupa.

En la carrera científica la cosa es diferente. Con un nivel de especialización cada vez mayor, es prácticamente imposible establecer criterios de valoración que juzguen la calidad del trabajo en sí. Con demasiada frecuencia, en el proceso de selección y de promoción, el candidato o candidata tiene un conocimiento más profundo de la materia de la que se trata que los propios miembros del tribunal que han de juzgar la propuesta.

Es necesario, entonces, establecer unos criterios generales de evaluación que sean objetivos y cuantificables y que tengan una aplicación general y aparentemente igualitaria. ¿Cómo se establecen estos criterios? En mi opinión más sobre la base de la forma que del fondo. 


\section{Una visión de género en el Área de Recursos Naturales}

Se utilizan para ello unos parámetros que, por un lado, están basados en estereotipos que como hemos dicho más arriba se ajustan mejor a una concepción masculina de la ciencia y, por otro, en un trato dispar de una líneas respecto a otras lo que marca una desigualdad entre disciplinas.

Hemos dicho varias veces que el área de Recursos Naturales es muy amplia. Podríamos decir que Recursos Naturales engloba muchas 'subáreas' diferentes; diversas por la naturaleza de las líneas de investigación que abordan, líneas que no son comparables unas con otras y, diversas también, por el diferente modo de entender como hacer Ciencia.

Es un área heterogénea. De ello da cuenta su producción científica que está, por el lado de las publicaciones con un mayor nivel de impacto en los puestos de cabeza de entre las del Organismo y, por otro, también está a la cabeza de las que publican más libros y asimilados lo que la coloca junto a otras áreas que se consideran menos competitivas. No tiene un papel menor en todo ello el hecho de que dentro del área hay disciplinas de moda y otras conceptuadas de anacrónicas. Me refiero a ellas no en un sentido peyorativo, pretendo únicamente contraponer dos enfoques de una situación por la que unas líneas de investigación o técnicas de trabajo tienen una mayor actualidad o atractivo y, en consecuencia, gozan de una mejor cabida en las revistas de más impacto. Esto, en demasiadas ocasiones, marca políticas científicas de apoyo a unas líneas en detrimento de otras.

Pero además es un área muy competitiva. El elevado número de publicaciones que la sitúan, como hemos dicho anteriormente, en el primer lugar entre las del CSIC si hablamos del número total de las producidas y la cantidad de recursos generados para investigación parece también avalar esta afirmación.

Amplitud, heterogeneidad y competitividad es el marco en el que hay que situar el problema de género que estamos abordando para tratar de explicar la situación tan atípica como peculiar del área.

No hay, en mi opinión, una explicación general que nos permita interpretar de modo único la situación de las investigadoras en las tres escalas pues, sin lugar a dudas, obedecen a causas y a circunstancias distintas. Circunstancias que no son ajenas a la propia evolución de las diferentes líneas de investigación y a coyunturas personales.

La cuestión de género en las escalas de Investigadores Científicos y Científicos Titulares sigue, en el área Recursos Naturales, la tónica más o menos general del CSIC con una desigualdad mayor pues los niveles están bastante por debajo de la media del Organismo.

Lo que requiere un análisis especial es el caso de las Profesoras de Investigación donde, como venimos apuntando, el porcentaje de un $22 \%$ 
está muy por encima de la media del Organismo que se sitúa en un 13,3\%. Adelanto una conjetura.

Es, por lo general, mediante un proceso de promoción interna como se accede a la escala de Profesores. El número de plazas ha sido muy escaso hasta ahora, lo que obliga a competir por una misma plaza a un buen número de investigadores de diferentes disciplinas científicas. Líneas de investigación, en suma, que no son comparables por la naturaleza de los trabajos que generan y la posibilidad de colocarlos en las revistas de mayor impacto. La disparidad de líneas no comparables es, en mi opinión, el primer factor discriminante. Solo los individuos cuyas líneas de investigación se adecuan mejor a los parámetros establecidos pasan la primera criba y sólo, en segundo lugar, está la discriminación de género. De tal modo que una investigadora que desarrolla su labor en una línea científica más en boga tiene mayores posibilidades de promoción que sus compañeros varones que trabajan en líneas menos competitivas. Podría ser ésta una de las causas que ayuden a explicar la situación atípica del área en este aspecto.

No olvidemos, en cualquier caso, que cuando estamos tratando con un número tan poco representativo como es el de ocho profesoras de investigación en un área con 306 investigadores pueden concurrir circunstancias personales, trayectoria científica o valía sobresaliente que no se pueden desestimar en absoluto, hasta tal punto que pueden trastocar por completo lo adecuado del anterior análisis a la realidad de los hechos.

\section{Colofón}

No hay una solución única al problema de género en el área, ni es un problema específico de Recursos Naturales, mas bien es una cuestión aún por solucionar hasta en los países económicamente más desarrollados (cf. Dewande, Science 295: 278-279. 2001). A problemas generales es deseable buscar soluciones generales pero, a veces, sólo es posible conseguir pequeños remedios parciales que, al complementarse unos con otros, ayuden a romper el círculo vicioso que la situación genera. Un estudio a fondo de la realidad de las investigadoras del CSIC, una mayor sensibilidad por parte de los estamentos de decisión de la Política Científica que se plasme en actuaciones concretas sería un primer paso en el largo camino que aún queda por recorrer. Hasta que ese momento llegue, dejamos algunas preguntas que, entre muchas otras, podrían servirnos de punto de partida a la reflexión desde dentro del CSIC: ¿En que proporción dirigen las mujeres equipos y/o proyectos de investigación? ¿Cuan- 


\section{Una visión de género en el Área de Recursos Naturales}

tas forman parte de comisiones de evaluación y tribunales de selección? $¿$ Cuantas dirigen o al menos forman parte de los estamentos del Organismo que rigen su política científica?

\section{Notas}

1 Pues se relacionan en el área una serie de Institutos que también se incluyen en la de Ciencias Agrarias, a saber: Ciencias Medioambientales (CCMA), Centro de Edafología y Biología Aplicada del Segura (CEBAS), Estación Experimental del Zaidín (EEZ), Instituto de Recursos Naturales y Agrobiología de Salamanca (IRNASA) y el Instituto de Recursos Naturales y Agrobiología de Sevilla (IRNASE). En el área de Tecnología de los Alimentos se incluye el Instituto de Agroquímica y Tecnología de los Alimentos (IATA) y, en él de Ciencias y Tecnologías Químicas, el Instituto de Productos Naturales y Agrobiología de Canarias (IPNA).

2 Los datos han variado ya que, hasta junio de 2001, el total de personal investigador se había incrementado en un $2 \%$ [cf.www.csic.es], incremento que en el área de Recursos Naturales ha sido del $4 \%$.

3 La fuente de datos consultada corresponde a las listas de personal investigador del Organismo hasta 1999.

\section{APÉNDICE I}

\begin{tabular}{|c|c|c|}
\hline Centro & Localidad & Temas de investigación \\
\hline $\begin{array}{l}\text { Centro de Estudios Avanzados de } \\
\text { Blanes (CEAB) }\end{array}$ & Blanes, Girona & $\begin{array}{l}\text { Sistemas litorales. Ecología de } \\
\text { poblaciones. Fauna y flora }\end{array}$ \\
\hline $\begin{array}{l}\text { Centro de Investigaciones sobre } \\
\text { Desertificación (CIDE) }\end{array}$ & Valencia & $\begin{array}{l}\text { Ciencias del suelo y procesos } \\
\text { superficiales }\end{array}$ \\
\hline $\begin{array}{l}\text { Estación Biológica de Doñana } \\
\text { (EBD) }\end{array}$ & Sevilla & $\begin{array}{l}\text { Ecología evolutiva y del } \\
\text { comportamiento. Ecología de } \\
\text { poblaciones }\end{array}$ \\
\hline $\begin{array}{l}\text { Estación Experimental de Zonas } \\
\text { Áridas (EEZA) }\end{array}$ & Almería & $\begin{array}{l}\text { Ciencias del suelo y procesos } \\
\text { superficiales. Ecología evolutiva y } \\
\text { del comportamiento. Ecología de } \\
\text { poblaciones }\end{array}$ \\
\hline $\begin{array}{l}\text { Instituto Andaluz de Ciencias de la } \\
\text { Tierra (IACT) }\end{array}$ & Granada & $\begin{array}{l}\text { Geoquímica. Estructura y génesis } \\
\text { minerales. Evolución y dinámica } \\
\text { de la litosfera }\end{array}$ \\
\hline Instituto de Astronomía y Geodesia & Madrid & Evolución y dinámica de la litosfera \\
\hline $\begin{array}{l}\text { Instituto de Acuicultura Torre de la } \\
\text { Sal (IATS) }\end{array}$ & Castellón & Acuicultura y pesquerías \\
\hline $\begin{array}{l}\text { Instituto Botánico de Barcelona } \\
\text { (IBB) }\end{array}$ & Barcelona & Fauna y flora \\
\hline
\end{tabular}




\begin{tabular}{|c|c|c|}
\hline Centro & Localidad & Temas de investigación \\
\hline Instituto de Ciencias del Mar (ICM) & Barcelona & $\begin{array}{l}\text { Evolución y dinámica de la litosfera. } \\
\text { Estructura y procesos oceánicos. } \\
\text { Sistemas litorales. Acuicultura y } \\
\text { pesquerías }\end{array}$ \\
\hline $\begin{array}{l}\text { Instituto de Ciencias Marinas de } \\
\text { Andalucía (ICMAN) }\end{array}$ & Cáfiz & $\begin{array}{l}\text { Sistemas litorales. Acuicultura y } \\
\text { pesquerías }\end{array}$ \\
\hline $\begin{array}{l}\text { Instituto de Ciencias de la Tierra } \\
\text { "Jaime Almera" (ICTJA) }\end{array}$ & Barcelona & $\begin{array}{l}\text { Geoquímica. Estructura y génesis } \\
\text { minerales. Evolución y dinámica de } \\
\text { la litosfera. Ciencias del suelo y } \\
\text { procesos superficiales }\end{array}$ \\
\hline $\begin{array}{l}\text { Instituto de Geología Económica } \\
\text { (IGE) }\end{array}$ & Madrid & $\begin{array}{l}\text { Geoquímica. Estructura y génesis } \\
\text { minerales. Evolución y dinámica de } \\
\text { la litosfera }\end{array}$ \\
\hline $\begin{array}{l}\text { Instituto de Investigaciones Marinas } \\
\text { (IIM) }\end{array}$ & Vigo & $\begin{array}{l}\text { Estructura y procesos oceánicos. } \\
\text { Sistemas litorales. Acuicultura y } \\
\text { pesquerías }\end{array}$ \\
\hline $\begin{array}{l}\text { Instituto de Investigaciones en } \\
\text { Recursos Cinegéticos (IREC) }\end{array}$ & Ciudad Real & $\begin{array}{l}\text { Ecología evolutiva y del } \\
\text { comportamiento. Ecología de } \\
\text { poblaciones }\end{array}$ \\
\hline $\begin{array}{l}\text { Instituto Mediterráneo de Estudios } \\
\text { Avanzados (IMEDEA) }\end{array}$ & Esporles, Mallorca & $\begin{array}{l}\text { Estructura y procesos oceánicos. } \\
\text { Sistemas litorales. Ecología evolutiva } \\
\text { y del comportamiento. Ecología de } \\
\text { poblaciones. Flora y fauna }\end{array}$ \\
\hline $\begin{array}{l}\text { Instituto Pirenaico de } \\
\text { Ecología (IPE) }\end{array}$ & Zaragoza & $\begin{array}{l}\text { Ciencias del suelo y proceso } \\
\text { superficiales. Ecología evolutiva y } \\
\text { del comportamiento. Ecología de } \\
\text { poblaciones. Fauna y flora }\end{array}$ \\
\hline $\begin{array}{l}\text { Museo Nacional de Ciencias } \\
\text { Naturales (MNCN) }\end{array}$ & Madrid & $\begin{array}{l}\text { Geoquímica. Estructura y génesis de } \\
\text { minerales. Evolución y dinámica de } \\
\text { la litosfera. Ciencias del suelo y } \\
\text { procesos superficiales. Ecología } \\
\text { evolutiva y del comportamiento. } \\
\text { Ecología de poblaciones. Fauna y } \\
\text { flora }\end{array}$ \\
\hline Real Jardín Botánico (RJB) & Madrid & Fauna y flora \\
\hline
\end{tabular}

Se relacionan en él, los Institutos del área de Recursos Naturales, la localidad donde se ubican y sus líneas prioritarias de investigación (Fuente: Plan de actuación del CSIC, 2000-2004. Aprobado por el Consejo Rector del CSIC en mayo de 2001) 